Signal \& Image Processing : An International Journal (SIPIJ) Vol.7, No.2, April 2016

\title{
AN INNOVATIVE MOVING OBJECT DETECTION AND TRACKING SYSTEM BY USING MODIFIED REGION GROWING ALGORITHM
}

\author{
G.Sharmila Sujatha and Prof. V.Valli Kumari \\ Department of Computer Science and Systems Engineering, Andhra University, \\ Visakhapatnam, AP, India
}

\begin{abstract}
The ultimate goal of this study is to afford enhanced video object detection and tracking by eliminating the limitations which are existing nowadays. Although high performance ratio for video object detection and tracking is achieved in the earlier work it takes more time for computation. Consequently we are in need to propose a novel video object detection and tracking technique so as to minimize the computational complexity. Our proposed technique covers five stages they are preprocessing, segmentation, feature extraction, background subtraction and hole filling. Originally the video clip in the database is split into frames. Then preprocessing is performed so as to get rid of noise, an adaptive median filter is used in this stage to eliminate the noise. The preprocessed image then undergoes segmentation by means of modified region growing algorithm. The segmented image is subjected to feature extraction phase so as to extract the multi features from the segmented image and the background image, the feature value thus obtained are compared so as to attain optimal value, consequently a foreground image is attained in this stage. The foreground image is then subjected to morphological operations of erosion and dilation so as to fill the holes and to get the object accurately as these foreground image contains holes and discontinuities. Thus the moving object is tracked in this stage. This method will be employed in MATLAB platform and the outcomes will be studied and compared with the existing techniques so as to reveal the performance of the novel video object detection and tracking technique.
\end{abstract}

\section{KEYWORDS}

Adaptive Median filter (AMF), Modified Region growing (MRG), Morphological Operation, Object Detection, Tracking.

\section{INTRODUCTION}

Object detection is done to find out the presence of objects in video frame and to detect those objects [12]. Then the object which is detected is categorized into various classes such as humans, vehicles and other moving objects [13]. Object tracking involves the process of segmenting a region of interest from video frames and tracking its motion and position [14]. Nowadays moving object tracking from the video sequences plays a vital role just because of its enormous practical applications like visual surveillance, perceptual user interface, content-based image storage and retrieval, athletic performance analysis etc [16]. But object tracking in DOI : $10.5121 /$ sipij.2016.7203 
Signal \& Image Processing : An International Journal (SIPIJ) Vol.7, No.2, April 2016

dynamic sequences like waving trees, fountains and oceans etc. still remains a tedious task [14]. Moving object detection includes video acquisition, object detection and tracking. Video surveillance is nothing but the process of examining video sequences [17], and also an effective area in computer vision, which yields large amount of storage and display. Video surveillance is broadly classified into three categories they are manual, semi-autonomous and fully-autonomous [13].

In security surveillance identifying a moving object, whether it is a human or non-human plays a vital role in most areas [19]. Human detection is a crucial area in research nowadays because of the rise in theft, terrorist and other illegal actions [20]. A structure which allows real time recognition of humans from a fixed camera makes the automatic video surveillance systems more dependable an efficient [10]. The three major steps in video analysis are detection, tracking and analyzing. (i.e. detecting the objects which are moving, tracking those objects frame by frame and analyzing the tracks of that object to identify their behavior). So the application of object tracking is suitable in tasks such as:

- Motion-based recognition, which means identification of humans on the basis of gait, automatic object detection etc;

- Automatic surveillance, which means observing a scene to identify dubious activities or improbable actions;

- Video indexing, which means automatic annotation and retrieving the videos in multimedia databases;

- Human-computer interaction, which means gesture recognition, eye gaze tracking for inputting the data into computers;

- Traffic monitoring, which means directing traffic flow by gathering real-time traffic statistics;

- Vehicle navigation, which means path planning based on videos and capability of avoiding obstacles.

For simple Object Detection and Tracking several techniques or algorithms are used. Although these techniques are implemented for different objectives like noise reduction or working on specific images (e.g. Gray scale images) or to diminish complications; etc; limitations are there while applying these algorithms or techniques. The techniques used nowadays for moving object detection are the frame subtraction method, the background subtraction method and the optical flow method [1, 2]. Frame subtraction is nothing but it takes the difference of two consecutive frames in order to identify the moving objects [13]. The computation for the frame subtraction method is uncomplicated and simple to develop. Moving Object Detection with Moving Camera is very difficult job[27]. Most of the algorithms focus independently to solve the object detection problem or the tracking problem [9]. Even though there are different algorithms available to solve all the problems, but the desirable option is to combine these algorithms so that an optimal solution could be obtained for the problems $[13,7]$.

The organization of this paper is arranged as follows. In section 2, a concise talk about the relevant research works are specified. In section 3, our novel object detection and tracking technique is described concisely. Section 4 includes the implementation results and performance analysis. Section 5 terminates the paper. 
Signal \& Image Processing : An International Journal (SIPIJ) Vol.7, No.2, April 2016

\section{RECENT RELATED RESEARCHES: A REVIEW}

Shuai Zhang et al. [21] projected an object detection technique by means of mean shift (MS) segmentation algorithm. The objects were again separated by using depth information which was derived from stereo vision. The objects which were detected from the above technique were then tracked by means of a novel object tacking algorithm with the help of a new Bayesian Kalman Filter (BKF) with the Simplified Gaussian Mixture (BKF-SGM). This BKF-SGM uses a GM representation of both static and noise densities. In order to avoid exponential complexity growth of conventional KFs with GM an innovative direct density simplifying algorithm is used. More robust tracking performance is achieved, when this BKF-SGM is coupled with an improved MS tracker, and the algorithm so obtained is called BKF-GSM-IMS algorithm.

Karthick Muthuswamy et al. [22] proposed a technique to generate spatial and motion saliency maps on the basis of comparison of local features and dominant features which are available in the frame. In this method salient region was marked if there was a huge variation between local and dominant features. Hue and Saturation features were used in spatial saliency and Optical flow vector features were used in motion saliency. Then the function was estimated by segmentation datasets. A simple algorithm was developed so as to create spatio-temporal saliency map which carry out a lot of state-of-the-art techniques.

Meenakshi Gupta et al. [23] proposed a technique called background subtraction method which has a combination of both depth segmentation detector and template matching technique so as to automatically initialize the human tracking. In order to the track the human silhouette in an active surrounding, when the robot was in motion, an innovative idea of head and hand creation on the basis of depth of interest was presented in that paper. A series of detectors (e.g. height, size and shape) was used in order to differentiate human from other moving objects so as to make this algorithm or technique powerful. As this silhouette-matching-based-method requires somewhat more calculating time the matching-based-method was used only when it was required. The location of humans in the frame was predicted by an unscented Kalman filter in order to sustain the stability of robotic motion.

Hailing Zhou et al. [24] presented proficient road detection and tracking framework in UAV videos. This road detection and tracking was done by two approaches they are graph-cut-baseddetection and homography-based road tracking. During the initial stage a graph-cut-baseddetection was used so as to extract a specific region in the road, and for tracking a fast homography-based road tracking was used in order to track the road areas automatically. Graphcut-based-detection was performed when there was a need to detect the road and generally most of the work was done by the fast homography-based road tracking.

Ahlem Walha et al. [25] proposed a system called video stabilization and moving object detection on the basis of camera movement evaluation. The global motion was calculated approximately with the help of local feature extraction and matching. For stabilization task they reveal the Scale Invariant Feature Transform (SIFT) key points. When the approximate calculation was done the global camera motion parameters uses affine transformation, where the moving objects are detected using Kalman filtering. A median filter was used to maintain the desired motion for motion smoothing. At the final point in order to get the stabilized video sequence, motion compensation was performed. The efficiency of the proposed system was revealed by numerous aerial video examples. The above system uses the software Virtual Dub 
Signal \& Image Processing : An International Journal (SIPIJ) Vol.7, No.2, April 2016

with Deshaker-plugin for testing purposes. For objective estimation they employ Interframe Transformation Fidelity and for the moving object detection task they utilize the Detection Ratio.

Bodhisattwa Chakraborty et al. [26] projected a technique called trajectory based ball-detectionand-tracking-method so as to detect and track the ball in a group of videos of basketball long shot sequences. The methodology used in the above paper is a two-fold detection and tracking framework, in the initial phase the detection of ball was done with the help of feature based method. The second phase involves the verification of the first stage, that is whether the detected object obtained in the first stage was ball or not, this was performed with the help of 2D trajectory information from the possible ball candidates in the frame. From the collection of candidate trajectories a proper ball trajectory was obtained and the ball locations were predicted from the obtained trajectories. Because of the occlusion of the ball and assimilation of the ball image with other objects from the background image ball positions were missed. These missed ball positions were predicted with the help of trajectory interpolation technique.

\section{THE PROPOSED MOVING OBJECT DETECTION AND TRACKING SYSTEM USING MODIFIED REGION GROWING METHOD}

Our proposed technique makes use of modified region growing algorithm for moving object detection. The object thus detected has been tracked with the help of multi features threshold value. We use video as an input which has been obtained from the database. Originally, the input video which has been obtained from the data base has split up into many frames.

Let $\left(V_{d}\right)$ be the video database and $\left\{\mathrm{v} 1_{\mathrm{i}, \mathrm{j}}, \mathrm{v} 2_{\mathrm{i}, \mathrm{j}} \ldots \mathrm{v} \mathrm{n}_{\mathrm{i}, \mathrm{j}}\right\}$ be the total number of frames in the video.

The frames thus obtained undergo preprocessing so as to eliminate the noise present in the frames. We have used an adaptive median filter in this preprocessing phase in order to get rid of noise. Segmentation has been done from the pre-processed image obtained from the above stage with the help of modified region growing algorithm. Subsequently, the background in the video frame has been subtracted by means of frame differencing method. From the segmented frame and its related background frame the multi-features like color, texture, motion, wavelet, edge has been taken out. The distance between background features and segmented features were computed by means of block matching algorithm and the result so obtained is compared with the threshold value. If it suits the threshold value it is considered as foreground object, morphological operations like opening and closing operations has been performed in the foreground object so as to eliminate the holes and unwanted breaks present in it.

Our proposed system deals with five phases they are,

i) Pre-processing

$\checkmark$ Adaptive median filter

ii) Object detection

Modified region growing algorithm 
Signal \& Image Processing : An International Journal (SIPIJ) Vol.7, No.2, April 2016

iii) Feature extraction
$\checkmark$ Color
$\checkmark$ Texture
$\checkmark$ Motion
$\checkmark$ Wavelet
$\checkmark$ Edge
$\checkmark$ Mean
$\checkmark$ Standard deviation
$\checkmark$ Skewness

iv) Hole filling

$\checkmark$ Morphological operation.

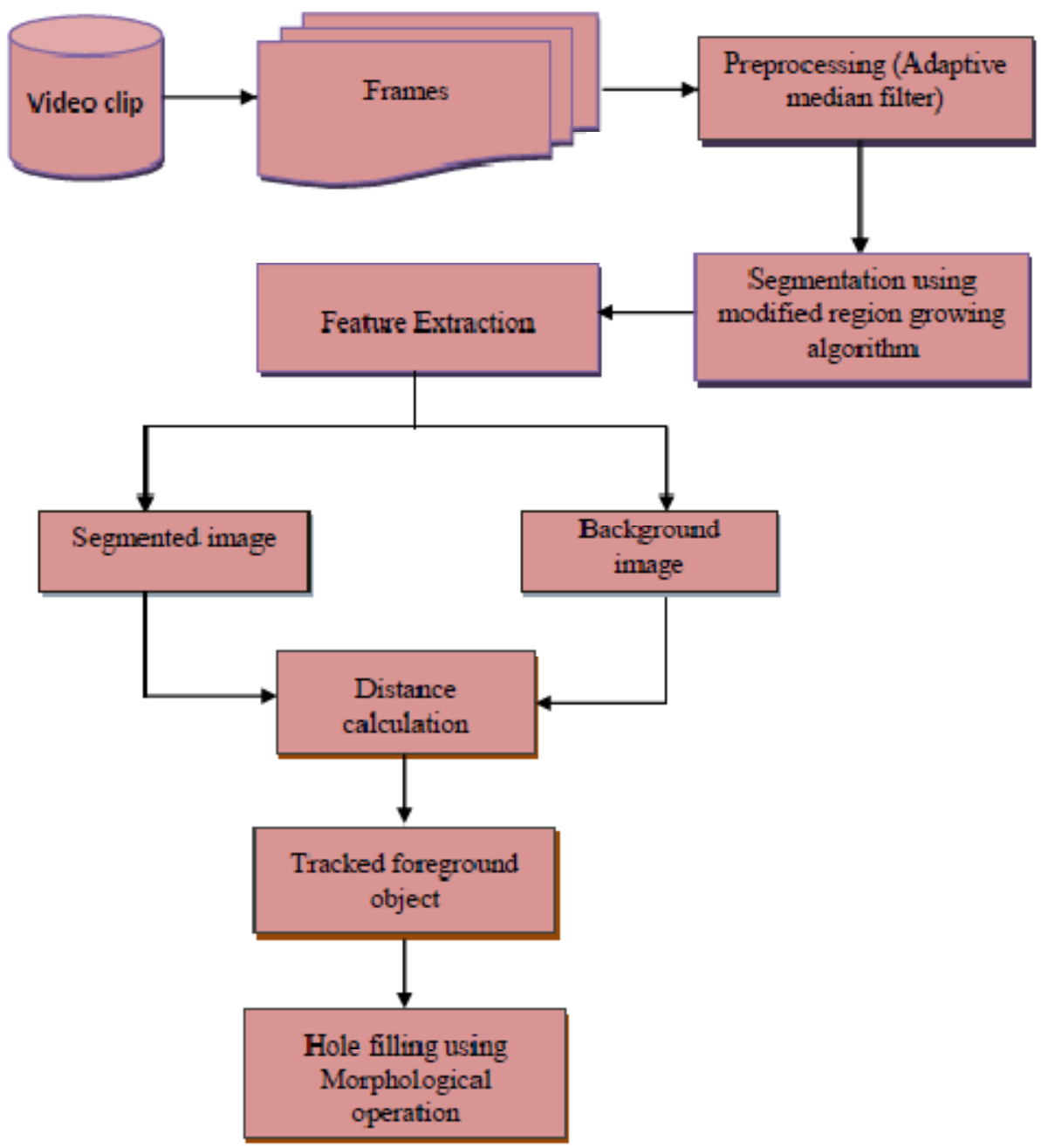

Figure1: Architecture of our proposed technique 
Signal \& Image Processing : An International Journal (SIPIJ) Vol.7, No.2, April 2016

\subsection{Preprocessing by utilizing Adaptive Median Filter (AMF)}

Pre-processing was done in the input frames by means of Adaptive Median Filter (AMF) in order to eliminate the noise present in the frames. Initially it detects the probable noisy pixels, subsequently it interchange them by means of its variants while the good pixels in the image were kept unaffected. The AMF filter performs well for revealing the noise still at a high noise level. $90 \%$ of the salt-and-pepper noise was eliminated by means of this filter. Noise removal from the input image should be made mandatory in order to attain better accuracy. Adaptive Median Filter performs based on the local statistics characters where the impulse was detected by manipulating the variation between the standard deviation of the pixels inside the filter window and the related current pixel.

Consider the video $\left(V_{d}\right)$ contains the video frame of $n$ numbers. Let $v_{i, j}$ be one of the grey level frame in the image $v$ at location $(i, j)$ taken from the video. Let $v_{\min }, v_{\max }$ are the lower and upper bounds of the image $v$.

$$
v_{\min } \leq v_{i, j} \leq v_{\max } \forall(i, j) \in a, a \equiv\{1,2, \ldots m\} \times\{1,2, \ldots n\}
$$

* The pixel location $(i, j)$ for the grey level image $v$ is given by the probability

$$
p_{i, j}=\left\{\begin{array}{l}
v_{\min }, \text { with probability } p_{x} \\
v_{\max }, \text { with probability } q_{x} \\
v_{i, j}, 1-p-q
\end{array}\right.
$$

The noise level, $\quad n(l)=p_{x}+q_{x}$

* $\quad v_{\min }, v_{\max }$, Local mean value $l(\mu)$ of the moving window and Local standard deviation $l(\sigma)$ are computed.

* Standard deviation and a user defined multiplier upper and lower bounds are computed subsequently with the help of local mean.

\subsection{Object detection:}

\subsubsection{Segmentation using modified region growing algorithm:}

For image segmentation region growing technique is a renowned method, where the segmentation is done based on seed point selection. Initially, in order to check whether the neighboring pixels in the image can be included with the region or not, the neighboring pixels are compared with the initial seed points. In segmentation process, the seed point selection plays a crucial role. The normal Region Growing Technique selects the seed points based on the intensity threshold, which fallout to over-segmentation or holes due to the imperfections in the image such as noise or deviation in intensity. And also by using the normal region growing technique the shadings of real images not get differentiated. In order to overcome these limitations, the intensity and orientation threshold features are included while selecting the seed points in the pre-processed images. The step by step procedure of MRG technique is illustrated 
below:

Step 1: Calculate the gradient of the frames for $x$ axis and $y$ axis, let it be $\left(\hat{g} x_{r d}\right)$ and $\left(\hat{g} y_{r d}\right)$.

Step 2: Integrate the gradient values based on the Equation shown below, so as to find the gradient vector $G V$

$$
G V=\frac{1}{1+\left(\hat{g} x_{r d}{ }^{2}+\hat{g} y_{r d}{ }^{2}\right)}
$$

Step 3: Attain the values of orientation by modifying the gradient vector values from radians to degrees.

Step 4: Segment the image into grids $\operatorname{grd}_{i}$.

Step 5: Ordain the intensity threshold as $\left(I_{(t h l d)}\right)$ and orientation threshold as $\left(O_{(t h l d)}\right)$.

Step 6: Continue the subsequent procedures in step 7 regarding each grid grd $_{i}$, until the number of grids reach the total number of grids for an image.

Step 7(a): Find out the histogram (hsgm) of each pixel in $\operatorname{grd}_{i}$.

step 7(b): Choose the most frequent histogram of the $\operatorname{grd}_{i}{ }^{\text {th }}$ grid and it denoted as Freq $q_{(h s g m)}$.

Step 7(c): Select any pixel, as per Freq $q_{(h s g m)}$ and assign that pixel as the seed point which has the intensity $\left(I y_{p}\right)$ and Orientation $\left(O n_{p}\right)$.

Step 7(d): The intensity and orientation of the neighboring pixel is considered as $\left(I y_{n}\right)$ and $\left(O n_{n}\right)$.

Step 7(e): Determine the intensity and orientation difference of the pixels $p$ and $n$ by using the equations given below.

$$
\begin{aligned}
d i f_{\text {int }} & =\left\|I y_{p}-I y_{n}\right\| \\
d i f_{\text {orient }} & =\left\|O n_{p}-O n_{n}\right\|
\end{aligned}
$$

Step 7(f): If $d i f_{\text {int }} \leq I_{(\text {thld })} \& \& d i f_{\text {orient }} \leq O_{\text {thld }}$, then make the region to grow by adding the related pixel to the region or else, go to step 7(h).

Step 7(g): Scrutinize whether all the pixels are added to the region. If yes, go to step 6, or else go to step 7(h). 
Signal \& Image Processing : An International Journal (SIPIJ) Vol.7, No.2, April 2016

Step 7(h): Re-examine the region and locate the new seed points and perform the procedure from step 7(a).

Step 8: Terminate the entire procedure.

Thus the moving object is segmented from the video frames by means of modified region growing algorithm.

\subsection{Feature extraction :}

The unique form of dimensional reduction is termed as feature extraction. If the input data of an algorithm for processing is more it is so called disreputably redundant. Transformation of the input data will be done so as to get the reduced representation set of features. Transforming the input data into the set of features is named as feature extraction. From the segmented and the background image the multi features such as color, texture, motion, wavelet, edge, mean standard deviation, and skewness were extracted during the feature extraction stage of our technique.

\subsubsection{Color feature}

For multimedia context and video retrieval, color is one of the important visual features . In our technique the color features are extracted by means of applying histogram on the video shots. This histogram provides the compact summary of the distribution of data in an image or video.

Initially re-sampling process is carried out for the image after that anisotropic diffusion process is carried out then for the anisotropic diffused image histogram is applied.

\section{Histogram}

Color histogram is nothing but a K-dimensional vector. Each component $h_{k}$ in this vector signifies the relative number of pixels of color $C_{k}$ in the image, which means the fraction of the pixels are nearly alike with the equivalent color. In order to construct the color histogram, transformation and quantization should be done. In the former, the image colors are transformed to the suitable color space and in the latter, quantization should be done based on a specific codebook of size K.

$$
I_{(h)} i=\frac{n_{o c r}}{N}, 0 \leq i<g l
$$

Where, $g l$ denotes the total number of gray levels in the image, $n_{\text {ocr }}$ denotes the number of occurrences of gray leveli. $N$ denotes the total number of pixels in the image, and $I_{(h)} i$ denotes the image histogram for pixel value $i$. 
Signal \& Image Processing : An International Journal (SIPIJ) Vol.7, No.2, April 2016

\subsubsection{Texture feature (LGXP)}

In our technique, Texture feature extraction is done by means of local gabor xor patten. LGXP operator is employed for the quantized phases of the central pixels and its neighbors, which is calculated from the equation mentioned below

$$
L G X P_{\mu, h}^{g}=q\left(\varphi_{\mu, h}\left(z_{c}\right) X O R q\left(\varphi_{\mu, h}\left(z_{i}\right)\right)\right.
$$

Where $L G X P_{\mu, h}^{g}=(K=1,2, \ldots, K)$ signifies the pattern calculated between $\varphi_{\mu, h}(i)$ and its neighbor $z_{c}, \varphi_{\mu, h}\left(z_{c}\right)$ denotes the phase, $q\left(\varphi_{\mu, h}\left(z_{i}\right)\right)$ denotes the quantized value of the phase and $\varphi_{\mu, h}(l)$ denotes the position of the central pixel in the Gabor phase map of scale $w$ and orientation $\mu, k$ denotes the neighborhood size.

At last the consequential binary labels are concatenated together so as to form a local pattern of the central pixel.

$$
L G X P_{\mu, h}\left(z_{c}\right)=\left[L G X P_{\mu, h}^{k}, L G X P_{\mu, h}^{k-1}, \ldots \ldots \ldots \ldots . . . . . L G X P_{\mu, h}^{1}\right]_{\text {binary }}=\sum_{i=1}^{k} 2^{i-1}, L X G P_{\mu, h}^{i}
$$

The outcome of the LGXP process is an equivalent value and the original value gets replaced by this equivalent value, this procedure is done for all blocks

\subsubsection{Motion feature extraction using block matching algorithm}

In a video sequence, the process of detecting the motion vectors which forms the transition from one frame to other is termed as motion estimation. Based on the motion of the object, we can be able to detect and track the object in any video. In our proposed technique, for motion estimation we have used the block matching algorithm.

\subsection{Object tracking using dissimilarity calculation}

During object tracking phase, the segmented image features and the background image features are compared and the dissimilarities between them will be computed. The outcome thus obtained is compared with the threshold value $(\eta)$. The equation given below clearly illustrates the dissimilarity calculation,

$$
\text { tracked object }=\left\{\begin{array}{l}
\text { correct },(\eta) \leq \text { disimilarity } \\
\text { not correct },(\eta)>\text { disimilarity }
\end{array}\right.
$$

An object is said to be tracked, when the object distance satisfies the threshold condition in the equation demonstrated above. The object thus tracked undergoes post processing. The holes and 
Signal \& Image Processing : An International Journal (SIPIJ) Vol.7, No.2, April 2016

the discontinuities elimination of the tracked object is done in the post processing stage by means of morphological operation.

\subsection{Post processing using morphological operation}

Images are performed. During opening, the original image is eroded and subsequently it undergoes dilation. During closing, the original The object tracked using dissimilarity calculation contains holes and discontinuities which are removed by means of morphological operations like erosion and dilation.

Erosion and dilation operations are nothing but the minimization and maximization value in the window. After performing the erosion and dilation operations, the intensity of the image get enhanced or it is brighter than the original image. Subsequently the image enlarges and shrinks (thins) by using these operations. The enlarged image is used frequently to fill the spaces. By means of the erosion and dilation operations the opening and closing of the image is dilated and subsequently it undergoes erosion.

\subsubsection{Operations of Opening and Closing}

The outcome of the opening operation is that it makes the shape of the object smooth, cuts off the thin, irregular and removes thin protrusions. In closing operation, it makes the shape of the object smooth; but in contrast to opening operation it eliminates the discontinuities and bridges the long, thin gap, gets rid of tiny holes and fills the cracks in the contour line.

\subsubsection{Erosion operation}

The erosion operation is one of the essential parts in morphological processing. In binary image the erosion process makes the object to "shrink" or "thin". The shrink operation is done by means of a structure element

\subsubsection{2 .Dilation Operation}

The dilation operation is also a necessary part in morphological processing. In binary image the dilation operation makes the object to "lengthen" or "thick". The "lengthening" or "thickening" process in dilation also done by means of a structure element likewise in erosion operation.

\section{EXPERIMENTAL RESULTS AND DISCUSSION}

Our novel technique of moving object detection and tracking system is employed in the MATLAB platform (version 14 a) with machine configuration as mentioned below:

Processor: Intel core i3

OS: Windows 7

CPU speed: $2.40 \mathrm{GHz}$

RAM: 4GB

The performance of our proposed technique of moving object detection and tracking system based on modified region growing technique and multi feature thresholding is assessed by means 
Signal \& Image Processing : An International Journal (SIPIJ) Vol.7, No.2, April 2016

of different videos. The fig 3 are the original images attained from shot segmentation process. Fig 4 are the preprocessed frames. Fig5 (a) shows the resultant images achieved during the object detection process and at last fig 6 (a) represents the tracked images in the video The sample images obtained from the video (1) and video (2) are shown in Fig.3 to 5.
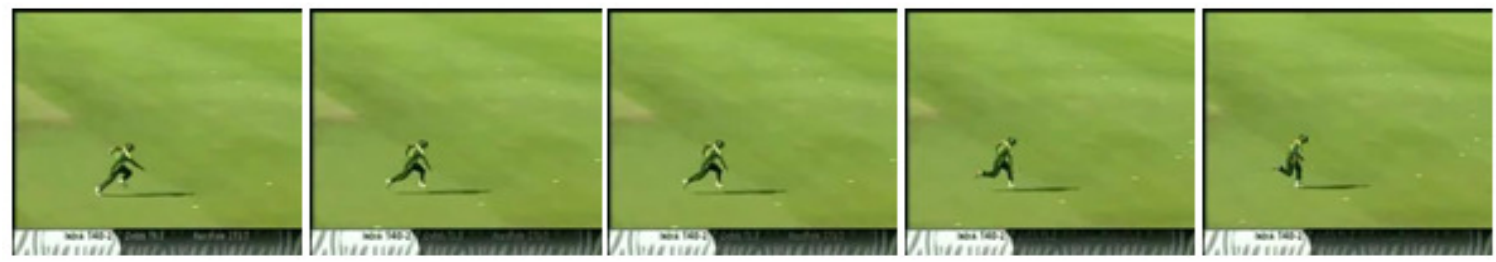

Figure 3 are the original frames obtained by means of shot segmentation process in video

In order to eliminate the noise from the above frames it undergoes preprocessing by means of an adaptive filter. Fig. 4 represents the frames obtained after noise removal in the preprocessing stage.
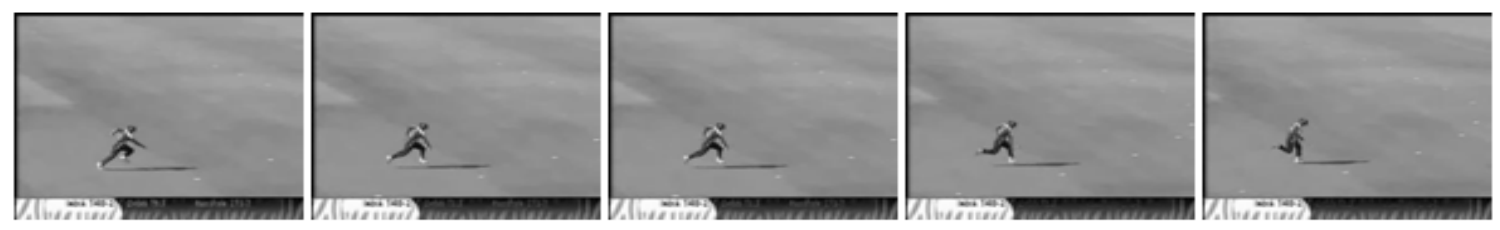

Figure 4 shows the pre-processed frames in video

Inorder to detect the object from the frames obtained above it then undergoes segmentation process by means of modified region growing algorithm. The segmented object obtained from the above frames are shown in fig 5 .
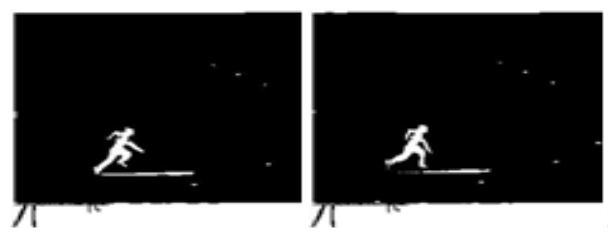
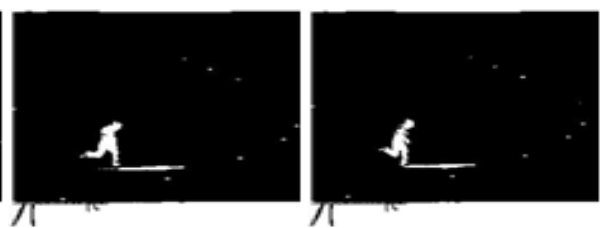

Fig 5

\subsection{Performance Analysis}

The performance of our proposed method of moving object detection and tracking system is studied by employing the statistical measures mentioned below.

$$
\begin{aligned}
\text { False Alarm Rate }(F A R) & =\frac{F P}{T P+F B} \\
\text { Detection Rate } & =\frac{T P}{T P+F N} \\
\text { Specificit } y & =\frac{T N}{F P+T N}
\end{aligned}
$$


Signal \& Image Processing : An International Journal (SIPIJ) Vol.7, No.2, April 2016

$$
\begin{array}{r}
\text { Acuuracy }=\frac{T P+T N}{T F} \\
\text { Position prediction }=\frac{T P}{T P+F P} \\
\text { Negative prediction }=\frac{T N}{F N+T N} \\
\text { False Negative Rate }=\frac{F N}{F N+T B} \\
\text { False Positive Rate }=\frac{F P}{F P+T N} \\
\text { TP } \times \text { TN }-F P \times F N
\end{array}
$$

\subsection{Comparative analysis:}

Comparative analysis includes the comparison of the proposed modified region growing algorithm with the existing region growing algorithm and fuzzy c means algorithm based on the conditions of statistical measures like accuracy, sensitivity, detection rate, NPV, PPV, FNR, FPR, MCC and FAR for the video the results obtained are shown in table.

\begin{tabular}{|c|c|c|c|}
\hline Measures & $\begin{array}{c}\text { Proposed modified } \\
\text { region growing }\end{array}$ & $\begin{array}{c}\text { Existing region } \\
\text { growing }\end{array}$ & Fuzzy C means \\
\hline Accuracy & 0.980149 & 0.9733639 & 0.9787655 \\
\hline Specificity & 0.848101 & 0.726918 & 0.771522 \\
\hline Detection Rate & 0.999475 & 0.978306 & 0.98289 \\
\hline NPV & 0.978239 & 0.994422 & 0.995388 \\
\hline PPV & 0.995752 & 0.402993 & 0.475434 \\
\hline FNR & 0.000525 & 0.021694 & 0.01711 \\
\hline FPR & 0.151899 & 0.273082 & 0.228478 \\
\hline MCC & 0.908562 & 0.529102 & 0.59548 \\
\hline FAR & 0.021761 & 0.005578 & 0.004612 \\
\hline
\end{tabular}

Table 1: Exemplifies the performance measures of the proposed moving object detection technique and the existing techniques of region growing algorithm and fuzzy c means for the video.

\section{Discussion}

Table 1 demonstrates the performance measures of our proposed moving object detection and tracking system for the video (2) which shows the improved accuracy, specificity and detection 
Signal \& Image Processing : An International Journal (SIPIJ) Vol.7, No.2, April 2016

rate of the proposed technique as when compared with the existing RGA and FCM techniques. This improved performance ratio indicates that our novel technique detects and tracks the object more accurately than the existing RGA and FCM techniques. The performance comparison graphs of accuracy, specificity and detection rate are exemplified in fig 6 .

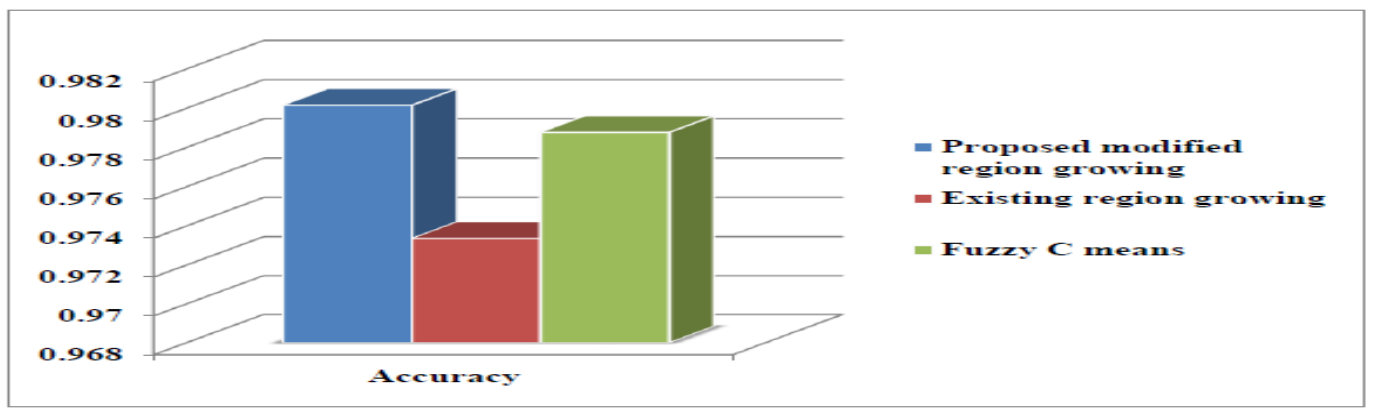

(i)

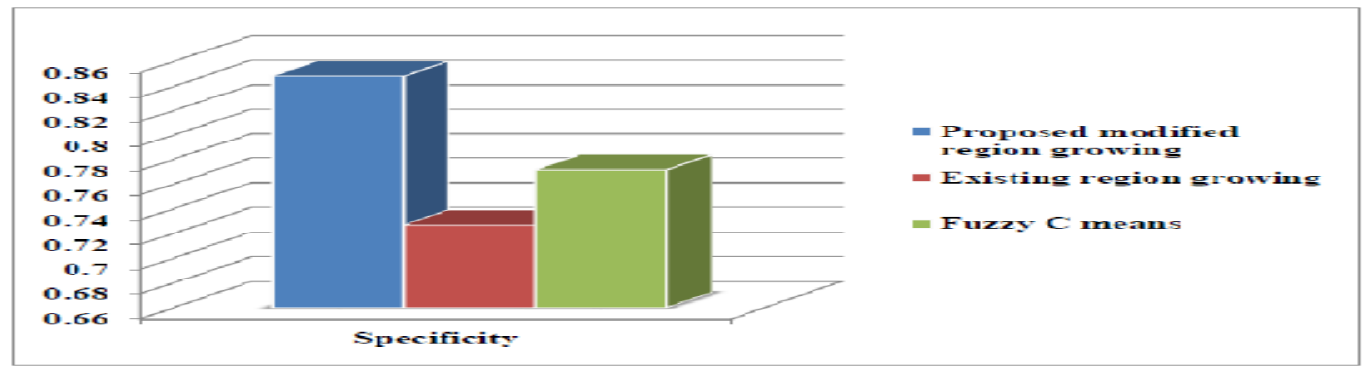

(ii)

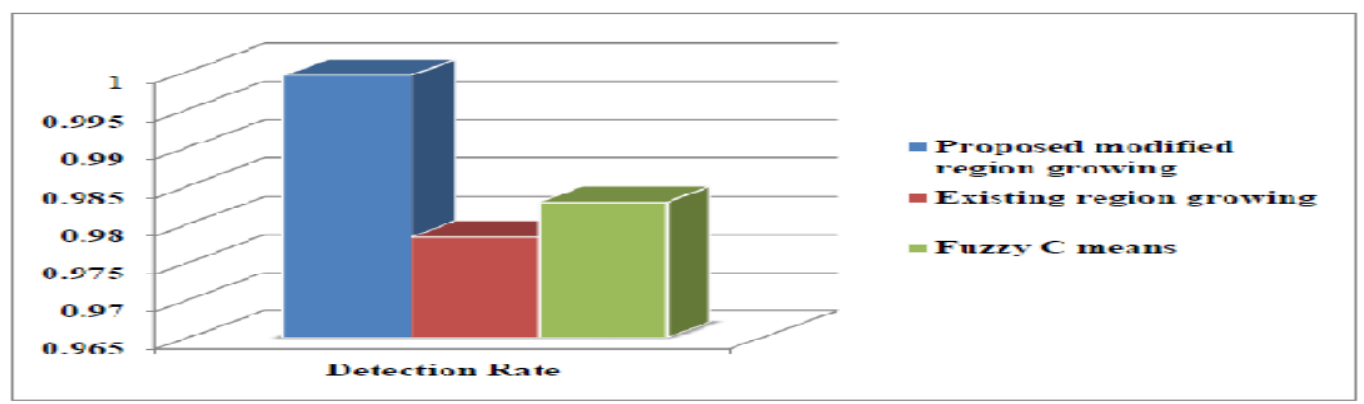

(iii)

Figure 6: Exemplifies the performance graph in terms of (i) Accuracy, (ii) Specificity and detection rate of moving object detection for the proposed technique and the existing techniques of modified region growing algorithm and fuzzy c means techniques for the video.

Moreover our proposed technique computational time is compared with the previous paper. And also with the existing techniques such as FCM and RGA and the comparison graph has been plotted below. 
Signal \& Image Processing : An International Journal (SIPIJ) Vol.7, No.2, April 2016

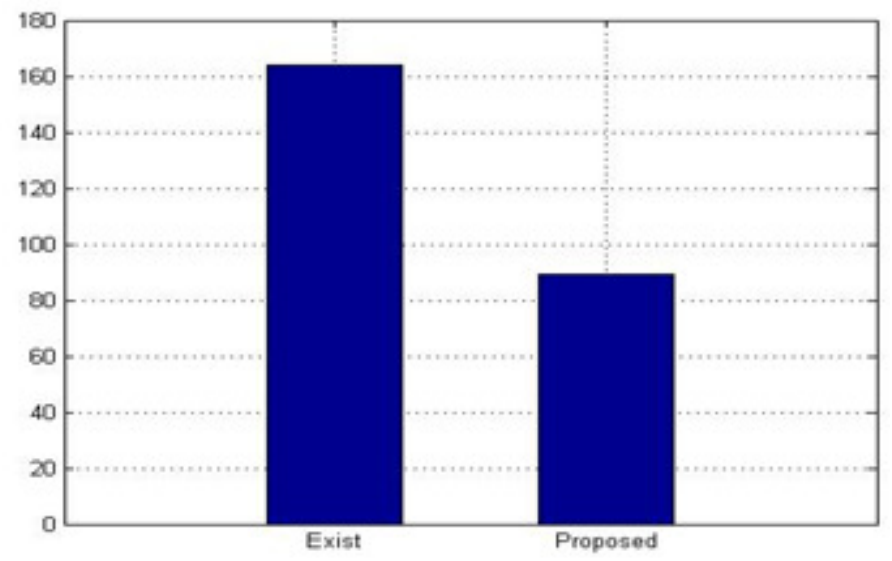

(i)

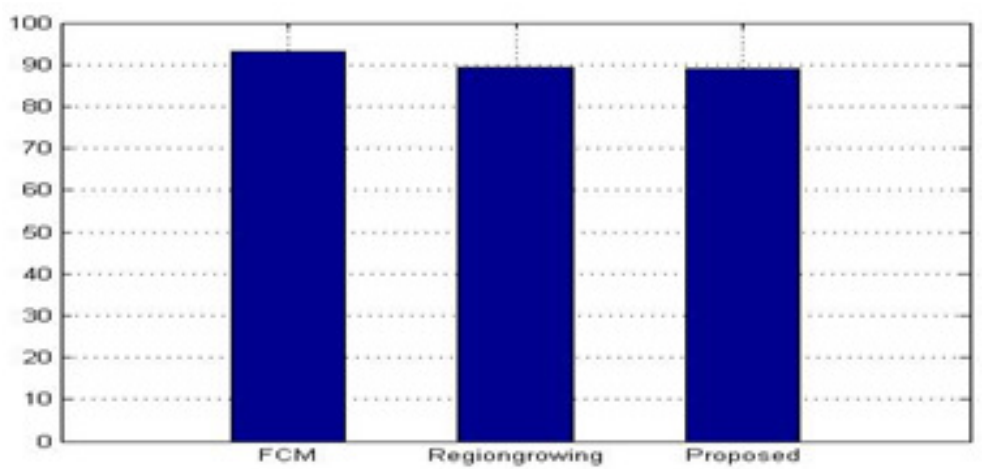

(ii)

Figure 7: shows the performance graph in terms of computational time

\section{Discussion:}

In the figure 7(i) our proposed technique is compared with the previous work. Similarly in figure 7(ii) our proposed technique is compared with existing algorithms such as FCM and Region growing. From the graph it has been clearly shown that our proposed technique consumes very low computational time than any other techniques. Hence it is proved that our proposed technique detects and tracks the object efficiently in low computational timing.

\section{CONCLUSION}

In this paper, we focus on the advancement in moving object detection and tracking system. In order to diminish limitations in the existing techniques, we have deliberately proposed moving object detection method by means of modified region growing technique. In comparative analysis, the performance of our technique is compared with the existing technique which shows that our novel moving object detection and tracking system yields improved performance. Moreover, the comparison results show that the accuracy and the computation time of our proposed method are better than the existing techniques. Consequently, it is proved that our proposed moving object detection and tracking system detects the object with more accuracy and proficiency and tracks the moving object with high performance and less computation time as when compared with the existing techniques. 
Signal \& Image Processing : An International Journal (SIPIJ) Vol.7, No.2, April 2016

\section{REFERENCES}

[1] Guler, Liang, Winnie Pushee and Ian, "A Video Event Detection and Mining Framework", In Computer Vision and Pattern Recognition Workshop, Conference on Madison, USA, Vol.4, pp.4242, 2003.

[2] Bhaskar and Suet-Peng Yong, "Image processing based vehicle detection and tracking method", In Computer and Information Sciences (ICCOINS), International Conference on Kuala Lumpur, pp.1-5, 2014.

[3] Pollard and Anton, "Detecting and tracking all moving objects in wide-area aerial video", In Computer Vision and Pattern Recognition Workshops (CVPRW), IEEE Computer Society Conference, pp.15-22, 2012.

[4] Rastegar, Babaeian, Bandarabadi and Toopchi, "Airplane detection and tracking using wavelet features and SVM classifier", In System Theory, 41st Southeastern Symposium, pp.64-67, 2009.

[5] Beihua, Xiongcai and Sowmya, "Robust human appearance matching across multi-cameras", International Conferencein Image Processing (ICIP), Melbourne, pp. 2222 - 2226, 2013.

[6] JiaweiHuang and Ze-Nian, "Automatic Detection of Object of Interest and Tracking in Active Video", 10th Pacific Rim Conference on Multimedia, Bangkok, Thailand,,pp 368-380, 2009.

[7] Varma and Sreeraj, "Object detection and classification in surveillance system", In Intelligent Computational Systems (RAICS), Trivandrum, pp. 19-21, 2013.

[8] Roomi, andKarpaga, "Detection and tracking of moving objects by fuzzy textures",Computing, Communications and Networking Technologies (ICCCNT),2013 Fourth International Conference on Tiruchengode, pp.1-5, 2013.

[9] Lu, Zhang, and Feng, "An efficient method for detecting ghost and left objects in surveillance video", In Advanced Video and Signal Based Surveillance, IEEE Conference on London, pp.540-545, 2007.

[10] Jiashu,and Heng-Ming Tai, "Efficient video object segmentation using adaptive background registration and edge-based change detection techniques", In Multimedia and Expo, IEEE International Conference on Taipei, Vol.2, pp.30-30, 2004.

[11] Ahmad, and Najam, "Video-based face classification approach: A survey", In Robotics and Artificial Intelligence (ICRAI), 2012 International Conference on Rawalpindi, pp. 179 - 186, 2012.

[12] Yun,and Song, Yanwei, Hui Tang, "Dynamic video object detection with single PTU camera", In Visual Communications and Image Processing (VCIP), pp. 1-4, 2011.

[13] Yuan, Xian sheng, Hongyu, and Jian Feng, "Detection and Tracking of Multiple Targets Based on Video Processing", In Intelligent Computation Technology and Automation, Second International Conference on Changsha, Hunan, pp. 586 - 589, 2009.

[14] Hariharakrishnan, Schonfeld, Raffy, and Yassa, "Video tracking using block matching", International Conferencein Image Processing (ICIP), Vol.3, pp.14-17, 2003.

[15] Payal Panchal, Gaurav Prajapati, Savan Patel, Hinal Shah, and Jitendra, "A Review on Object Detection and Tracking", International journal for research in emerging science and technology, Vol. 2,No. 1, pp. 7-12, 2015. 
Signal \& Image Processing : An International Journal (SIPIJ) Vol.7, No.2, April 2016

[16] Kinjal, and Darshak, "A Survey on Moving Object Detection and Tracking in Video Surveillance System”, International journal of soft computing and Engineering, Vol. 2, No-3, pp .44-48, 2012.

[17] Barga, and Dalton, "A Survey onMoving Object Tracking in Video", International journal of Information theory, Vol.3, No.3, pp.31-46, 2013.

[18] Jyoti Jadhav, "Moving Object Detection and Tracking for Video Surveillance", International journal of engineering research and general science, Vol.2, No.4, pp.372-378, 2014.

[19] Arunachalam, Sorimuthu, Rajagopal, and Sankaragomathi, "Automatic Fast Video Object Detection and Tracking on Video Surveillance System", Journal of image and video processing, Vol. 03, pp.479-484, 2012.

[20] Tushar, Yogendra and Kumar Jain, "Detecting and Tracking of Moving Objects from Video", International journal of computer application, Vol. 81, No.18, pp.23-28 2013.

[21] Shuai Zhang, Chong Wang, Shing-Chow Chan, Xiguang Wei and Check-Hei Ho, "New Object Detection, Tracking, and Recognition Approaches for Video Surveillance Over Camera Network", In Sensors Journal , Vol.15, No.5, pp.2679-2691, 2015.

[22] Muthuswamy, and Rajan, "Particle filter framework for salient object detection in videos", In Computer Vision, Vol. 9, No.3, pp.428-438, 2015.

[23] Gupta, Behera, Subramanian and Jamshidi, "A Robust Visual Human Detection Approach With UKF-Based Motion Tracking for a Mobile Robot", In Systems Journal, IEEE , Vol.9, No.4, pp.1363$1375,2015$.

[24] Hailing Zhou, Hui Kong, Lei Wei, Creighton, and Nahavandi, "Efficient Road Detection and Tracking for Unmanned Aerial Vehicle", IEEE Transactions in Intelligent Transportation Systems, Vol.16, No.1, pp.297-309, 2015.

[25] Ahlem walha, Ali wali and Adel .Alimi, "Video stabilization with moving object detecting and tracking for aerial video surveillance", Multimedia tools and application,Vol. 74, No. 17, pp. 67456767, 2015.

[26] Bodhisattwa and Sukadev, "A real-time trajectory-based ball detection and tracking framework for basketball video", Multimedia tools and application, Vol. 74, No.3, pp. 729-742, 2013.

[27] G.Sharmila Sujatha and Prof. V.Valli Kumari, "An Efficient Motion Based Video Object Detection and Tracking System", International Conference on Innovative Trends in Electronics Communications and Applications" (ICIECA15), vol. 1, No 13, pp85-97,2015.

\section{AUTHORS}

Prof. Valli Kumari , Deptartment of CS\&SE,College of Engg,.Andhra University She has total Teaching Experience 24 years.she also Honorary Director, Andhra University Computer Centre. She got Gold Medal for best Research (PhD thesis in Engineering:2004-2005), awarded at 75th Convocation (Diamond Jubilee) of Andhra University, January 2008. Best Paper award,INDICON-2011(IEEE International Conference held at BITS PILANI, Hyderabad campus, December, 2011) ,more than 60 papers published in conferences and journals, she had more than five editorial memberships, she is member of more than 20 reviewing

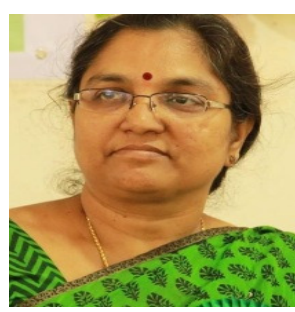
committe/technical committes. she is author of Privacy Preserving Publishing: A compilation of state of 
Signal \& Image Processing : An International Journal (SIPIJ) Vol.7, No.2, April 2016

the art works for researchers, OO Programming in Java, 2001, for School for Distance Education, Andhra University, Software Engineering, Printed and circulated, by CSE Association, , S.R.K R Engineering College ,1994,She is member of IEEE,ACM ,IETE more than this. She Two international level chapters.

G. Sharmila Sujatha working Assistant Professor (c ) in Dept of CS\&SE,Andhra University since 2001, Research area is Image Processing.Ten workshop/seminors attended,Three international Papers,Two international journals and Two national level papers are published.

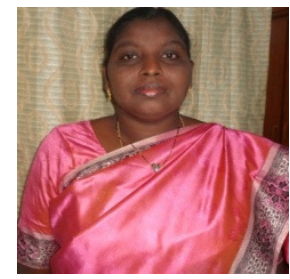

